\title{
Static Finite Particle Method and Geomechanics Application
}

\author{
Wenxiong Huang ${ }^{\text {i) }}$ and Ding Chen ${ }^{\text {ii) }}$
}

i) Professor, College of Mechanics \& Materials, Hohai University, 8 West Focheng Rd, Nanjing 211100, China.
ii) Ph.D Student, College of Mechanics \& Materials, Hohai University, 8 West Focheng Rd, Nanjing 211100, China.

\begin{abstract}
Like Smoothed Particle Hydrodynamics, the Finite Particle Method is conventionally formulated for dynamic problems and suitable for simulating dynamic motion of liquids and solids. In this work, the FPM is formulated as equilibrium problems large deformation of solids. Numerical aspects for implementation, including solution method, control of numerical instability, stress integration, are discussed. Application of the method to slope stability analysis is presented to demonstrates the effective, accuracy and the potential in solving large deformation and failure flow problems.
\end{abstract}

Keywords: FPM, static deformation, numerical implementation, slope stability analysis

\section{INTRODUCTION}

The Finite Particle Method (FPM) discussed in this paper is a collocational smoothed particle method developed on the basis of Smoothed Particle Hydrodynamic (SPH) method (Lucy, 1977; Monaghan, 1994). The FPM formulation was developed to overcome the boundary deficiency problem (Chen et al, 1999; Liu \& Liu, 2006). Similar to the SPH, the FPM is conventionally formulated for dynamic problems, and the governing equations are usually integrated explicitly for numerical efficiency. With special techniques for overcoming tension instability (Bonet \& Kulasegaram, 2001; Chen et al, 2019), the FPM can also be applied to simulate dynamic motion of solids. But the method is usually not suitable for solving static deformation and static flow of failure problems. Moreover, the dynamic procedure is not applicable to slope stability analysis, for which a limit state should be sought. In this work, the FPM method is formulated as an equilibrium problem for static deformation of solids. Numerical aspects for implicit integration of the governing equations are discussed. Application of the method to slope stability analysis is presented.

\section{FORMULATION OF FPM}

\subsection{Particle approximation}

The present smoothed particle method (FPM) is developed based on the particle discretization of the continuum body in deformation and simultaneous particle approximations for the displacement $u_{i}$ and the displacement gradient $u_{i, j}=\partial u_{i} / \partial x_{j}$, as in the following form:

$$
u_{i}^{a}=\sum_{b=1}^{n s} h_{0}^{a, b} u_{i}^{b} V^{b} ; \quad u_{i, j}^{a}=\sum_{b=1}^{n s} h_{j}^{a, b}\left(u_{i}^{b}-u_{i}^{a}\right) V^{b} .
$$

Herein the superscripts " $a$ " and " $b$ " indicate the quantities whose values are associated with the respective particles, $u_{i}^{a}=u_{i}\left(x_{i}^{a}\right), u_{i, j}^{a}=u_{i, j}\left(x_{i}^{a}\right) ; V^{b}$ represents the volume associated with particle $b$. Summation is carried over all particles in the influence domain of particle $a . h_{0}^{a, b}$ and $h_{j}^{a, b}(j=1,2,3)$ are the auxiliary kernels associated to particle " $a$ " and " $b$ ", which can be expressed as

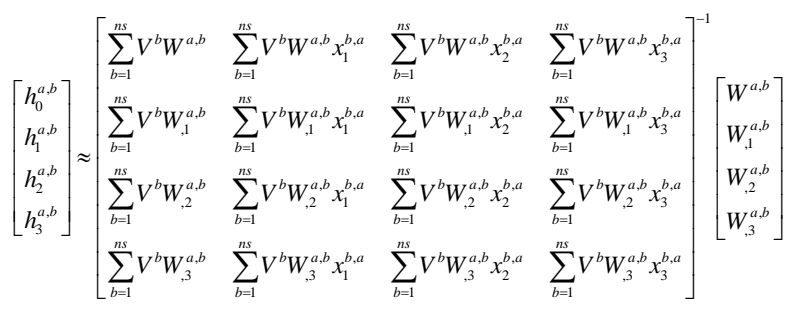

where $x_{k}^{b, a}=x_{k}^{b}-x_{k}^{a}$ represents difference of position vectors of particle $a$ and $b, W^{a, b}=W\left(x_{k}^{a}-x_{k}^{b}, l_{s}\right)$ and $W_{, j}^{a, b}=W_{, j}\left(x_{k}^{a}-x_{k}^{b}, l_{s}\right)$ are true kernel function and its partial derivatives.

\subsection{FPM governing equations for static problems}

The static deformation problem of a continuum body can be described by the virtual displacement equation: 


$$
\int_{\Omega} \delta \boldsymbol{\varepsilon}^{\mathrm{T}} \boldsymbol{\sigma} \mathrm{d} V=\int_{\Gamma_{\sigma}} \delta \mathbf{u}^{\mathrm{T}} \overline{\mathbf{t}} \mathrm{d} A+\int_{\Gamma_{u}} \delta \mathbf{u}^{\mathrm{T}} \lambda(\overline{\mathbf{u}}-\mathbf{u}) \mathrm{d} A+\int_{\Omega} \delta \mathbf{u}^{\mathrm{T}} \mathbf{b} \mathrm{d} V
$$

Here the matrix representations are used for Cauchy stress $\boldsymbol{\sigma}$, displacement $\mathbf{u}$, virtual strain $\delta \boldsymbol{\varepsilon}$, body force $\mathbf{b}$, surface traction $\overline{\mathbf{t}}$, etc.; $\Gamma_{u}$ and $\Gamma_{\sigma}$ are respectively the displacement boundary and surface traction boundary of the current configuration $\Omega ; \overline{\mathbf{u}}$ represents the prescribed displacement and $\lambda$ the Lagrangian multiplier for enforcing the displacement boundary condition. Through particle discretization, the displacements and their spatial gradients are represented by particle displacement as given in Eq.(1). It can be derived that the equilibrium of the particle system is governed by the following equation:

$$
\mathbf{F}(\mathbf{d}, t)=\mathbf{F}^{\mathrm{int}}-\mathbf{F}^{\mathrm{ext}}=\mathbf{0}
$$

with

$$
\begin{aligned}
& \mathbf{F}^{\mathrm{int}}=\sum_{a=1}^{n p} \mathbf{c}^{a \mathrm{~T}} \mathbf{H}_{\varepsilon}^{a \mathrm{~T}} \boldsymbol{\sigma}^{a} V^{a}+\sum_{c=1}^{n p 2} \mathbf{c}^{c \mathrm{~T}} \lambda^{c} \mathbf{H}^{c \mathrm{~T}}\left(\overline{\mathbf{u}}^{c}-\mathbf{H}^{c} \hat{\mathbf{d}}^{c}\right), \\
& \mathbf{F}^{\mathrm{ext}}=\sum_{c=1}^{n p 1} \mathbf{c}^{\mathrm{T} T} \mathbf{H}^{c \mathrm{~T}} \overline{\mathbf{t}}^{c} A^{c}+\sum_{a=1}^{n p} \mathbf{c}^{\mathbf{c}^{\mathrm{T}} \mathrm{T}} \mathbf{H}^{a \mathrm{~T}} \mathbf{b}^{a} V^{a} .
\end{aligned}
$$

Herein $\mathbf{H}^{a}$ and $\mathbf{H}_{\varepsilon}^{a}$ are matrices associated with particle $a$ and formed by auxiliary kernels $h_{0}^{a, b}$ and $h_{j}^{a, b}(j=1,2,3) \quad(b=1,2, \cdots, n s)$ respectively, with $n s$ being number of the particle in the influence domain of particle $a ; \hat{\mathbf{d}}^{a}$ is a column matrix containing displacement components of particles in the influence domain of particle $a$ arrayed in a local order; $\mathbf{c}^{a}$ is the selective matrix for $\hat{\mathbf{d}}^{a}$ to form the column matrix of the global displacement $\mathbf{d}$.

\section{NUMERICAL ASPECTS FOR IMPLEMENTATION}

\subsection{Solution method of the nonlinear system}

A static loading process described with a fictitious time $t \in[0, T]$ can be divided into a number of increment steps. The displacement will be integrated implicitly over the time domain so that equilibrium of the particle system holds at the end of each loading increment step. For a typical time increment step $\left[t_{n}, t_{n+1}\right]$ with known displacement $\mathbf{d}_{n}$, equilibrium of the particle system at the end of the step requires that

$$
\mathbf{F}\left(\mathbf{d}_{n+1}, t_{n+1}\right)=\mathbf{F}\left(\mathbf{d}_{n}, t_{n}\right)+\mathbf{J}\left(\mathbf{d}_{n}\right) \Delta \mathbf{d}+o\left(\|\Delta \mathbf{d}\|^{2}\right)=\mathbf{0}
$$

Herein $\Delta \mathbf{d}=\mathbf{d}_{n+1}-\mathbf{d}_{n}$ is the displacement increment to be determined; $\mathbf{J}=\partial \mathbf{F} / \partial \mathbf{d}$ is the Jacobi matrix. A Newton iteration scheme can be employed to solve the above nonlinear equation system:

$$
\left\{\begin{array}{l}
\mathbf{J}\left(\mathbf{d}_{n+1}^{k}\right) \delta \mathbf{d}^{k}=-\mathbf{F}\left(\mathbf{d}_{n+1}^{k}, t_{n+1}\right) ; \\
\Delta \mathbf{d}^{k+1}=\Delta \mathbf{d}^{k}+\delta \mathbf{d}^{k}, \quad \mathbf{d}_{n+1}^{k+1}=\mathbf{d}_{n}+\Delta \mathbf{d}^{k+1} .
\end{array}\right.
$$

The initial guess for the iteration can be set to $\Delta \mathbf{d}^{0}=\mathbf{0}$ and $\mathbf{d}_{n+1}^{0}=\mathbf{d}_{n}$.

\subsection{Hourglass control}

Most collocational meshless methods, including SPH and FPM, suffer from a numerical instability problem known as hourglassing. Due to rank deficiency of stiffness matrix, spurious deformation mode may enter to the numerical solution leading to an incorrect result. Various methods (Monaghan, 1994; Ganzenmüller et al, 2016; Vidal, 2007) have been proposed to handle this problem. In this work, the artificial viscosity as proposed by Monaghan (1994) is employed to control the hourglassing phenomenon. Between each pair of particles in interaction, a viscous force pair is applied. Thus an additional force of the following form will be applied to each particle:

$$
f_{i}^{\mathrm{va}}=V^{a} \sum_{b=1}^{n s}-V^{b}\left(\Pi^{a b} h_{i}^{a, b}-\Pi^{b a} h_{i}^{b, a}\right)
$$

Herein $\Pi^{a b}$ is the viscous force factor as given in 2), which is proportional to the relative velocity of particle $a$ and $b$, namely $\mathbf{v}^{\text {ab }}$. Adapted to the static problem, this relative velocity is approximated by

$$
\mathbf{v}^{\mathrm{ab}} \approx\left(\Delta \mathbf{u}^{b}-\Delta \mathbf{u}^{a}\right) / \Delta t
$$

Consequently, a pseudo-viscous force vector of the following form will be added to the global particle system:

$$
\mathbf{F}^{\mathbf{v}}\left(\mathbf{d}_{n+1}, \Delta \mathbf{d}\right)=\sum_{a=1}^{n p} \mathbf{c}^{a \mathrm{~T}} \mathbf{f}^{\mathrm{v} a}=\mathbf{k}^{v}\left(\mathbf{d}_{n+1}\right) \Delta \mathbf{d}
$$

This pseudo-viscous force is linear in displacement increment $\Delta \mathbf{d}$. The details of the matrix $\mathbf{k}^{v}\left(\mathbf{d}_{n+1}\right)$ is omitted.

It may be noted that introducing of the viscous force to the particle system does not change the global equilibrium of the system. With the viscous force added, the New iteration scheme (7) is modified to the following form:

$$
\left\{\begin{array}{l}
{\left[\mathbf{J}\left(\mathbf{d}_{n+1}^{k}\right)+\mathbf{k}^{v}\left(\mathbf{d}_{n+1}^{k}\right)\right] \delta \mathbf{d}^{k}=-\mathbf{F}_{n+1}^{k}-\mathbf{F}_{n+1}^{v k} ;} \\
\Delta \mathbf{d}^{k+1}=\Delta \mathbf{d}^{k}+\delta \mathbf{d}^{k}, \quad \mathbf{d}_{n+1}^{k+1}=\mathbf{d}_{n}+\Delta \mathbf{d}^{k+1} .
\end{array}\right.
$$

Herein $\quad \mathbf{F}_{n+1}^{k}=\mathbf{F}\left(\mathbf{d}_{n+1}^{k}, t_{n+1}\right), \mathbf{F}_{n+1}^{v k}=\mathbf{F}^{v}\left(\mathbf{d}_{n+1}^{k}, \Delta \mathbf{d}^{k}\right)$. The iteration terminates at meeting the conditions for convergence:

$$
\frac{\left\|\mathbf{F}_{n+1}^{k+1}+\mathbf{F}_{n+1}^{v k}\right\|}{\left\|\mathbf{F}_{n+1}^{0}\right\|}<\varepsilon_{\mathrm{F}} \quad \& \quad \frac{\left\|\delta \mathbf{d}^{k}\right\|}{\left\|\mathbf{d}_{n+1}^{k}\right\|}<\varepsilon_{\mathrm{u}},
$$

where $\varepsilon_{F}$ and $\varepsilon_{u}$ are prescribed error tolerances. 


\subsection{Tension instability elimination}

To eliminate the tension instability used to occur in the updated Lagrangian FPM for solids, Chen et al. (2019) introduced a technique, which permits the influence domain of each particle to deform with the continuum body, so that the same set of the particles is kept in the particle influence domain. This is realized by updating the auxiliary kernels at each increment step according to the following formulas:

$$
h_{0, n+1}^{a, b}=\alpha^{b} h_{0, n}^{a, b} ; \quad \mathbf{h}_{n+1}^{a, b}=\beta^{a b} \sum_{k=1}^{3} \mathbf{h}_{n}^{a, b}\left(\Delta \mathbf{F}_{n+1 / 2}^{a}\right)^{-1} .
$$

Herein $\mathbf{h}^{a, b}=\left[h_{1}^{a, b}, h_{2}^{a, b}, h_{3}^{a, b}\right]^{T}, \Delta \mathbf{F}_{n+1 / 2}^{a}=\mathbf{I}+\nabla_{n+1 / 2}\left(\Delta \mathbf{u}^{a}\right)$ is the increment deformation gradient associated to particle $a$, the transformation factors $\alpha^{b}$ and $\beta^{a b}$ are given as

$$
\alpha^{b}=\frac{V_{n}^{b}}{V_{n+1}^{b}} ; \quad \beta^{a b}=\frac{\left(\mathbf{x}_{n+1}^{b a}\right)^{T} \Delta \mathbf{F}_{n+1 / 2}^{a} \mathbf{x}_{n}^{b a}}{\alpha^{b}\left(\mathbf{x}_{n+1}^{b a}\right)^{T} \mathbf{x}_{n}^{b a}} .
$$

\subsection{Incrementally objective integration of stress}

On each time increment step, stress and other state variables should be updated according to the constitutive model to be implemented. As an example, implementation of an hypoelastic-plastic model of is discussed here. The constitutive model bounds the Cauchy stress tensor $\boldsymbol{\sigma}$ and the hardening variable $\mathbf{q}$ by the condition

$$
f(\boldsymbol{\sigma}, \mathbf{q}) \leq 0 .
$$

The relation between the objective stress rate $\stackrel{\sigma}{\sigma}$ and the deformation rate $\dot{\boldsymbol{\varepsilon}}$ is in the form of

$$
\dot{\boldsymbol{\sigma}}=\mathbf{D}:\left(\dot{\boldsymbol{\varepsilon}}-\dot{\boldsymbol{\varepsilon}}^{p}\right),
$$

where $\mathbf{D}$ is the elastic tensor and $\dot{\boldsymbol{\varepsilon}}^{p}$ the plastic part of the deformation rate tensor, which is determined by the flow rule:

$$
\dot{\boldsymbol{\varepsilon}}=\lambda \mathbf{m}(\boldsymbol{\sigma}, \mathbf{q}) \text {. }
$$

Herein $\mathbf{m}$ is a known tensorial function; the non-negative factor $\lambda$ can be determined from the consistency condition for plastic loading.

For solved particle displacement increment $\Delta \mathbf{u}^{a}$ on the increment step $\left[t_{n}, t_{n+1}\right]$, with the values for $\boldsymbol{\sigma}_{n}$ and other state variables having been determined in the previous step, the stress can be updated using the incrementally objective integration proposed by Hughes and Winget (1980) as the following.

First, calculate the effective deformation rate tensor and the effective spin tensor as

$$
\begin{aligned}
& \Delta \boldsymbol{\varepsilon}=\frac{1}{2}\left[\left(\nabla_{n+1 / 2} \Delta \mathbf{u}\right)+\left(\nabla_{n+1 / 2} \Delta \mathbf{u}\right)^{\mathrm{T}}\right], \\
& \Delta \boldsymbol{\omega}=\frac{1}{2}\left[\left(\nabla_{n+1 / 2} \Delta \mathbf{u}\right)-\left(\nabla_{n+1 / 2} \Delta \mathbf{u}\right)^{\mathrm{T}}\right]
\end{aligned}
$$

Then the orthogonal tensor

$$
\mathbf{Q}=\left(\mathbf{I}-\frac{1}{2} \Delta \boldsymbol{\omega}\right)^{-1} \cdot\left(\mathbf{I}+\frac{1}{2} \Delta \boldsymbol{\omega}\right)
$$

describes the rotation of the material element. The stress can be updated according to

$$
\boldsymbol{\sigma}_{n+1}=\mathbf{Q} \cdot \boldsymbol{\sigma}_{n} \cdot \mathbf{Q}^{\mathrm{T}}+\mathbf{D}: \Delta \boldsymbol{\varepsilon}-\lambda \mathbf{D}: \mathbf{m} .
$$

Implicit or explicit return mapping iterative scheme can be employed for the case of $\lambda>0$.

\section{SLOPE STABILITY ANALYSIS}

As an example of application in this work, the numerical procedure is developed for slope stability analysis. For this purpose, an elasto-perfectly-plastic model based on the Mohr-Coulomb failure criterion is implemented, which contains $E, v$ as elastic material parameters and $c, \phi$ as strength parameters. Limit state of the slope is sought through repeated application of FPM procedure using the constitutive model with reduced strength parameters:

$$
c^{*}=c / F_{R}, \quad \phi^{*}=\tan ^{-1}\left(\tan \phi / F_{R}\right) .
$$

The strength reduction factor is then used as the safety factor for stability assessment.

A uniform geo-slope of $1: 1$ gradient and $0.4 \mathrm{~m}$ height, subjected to gravity, is considered. The values of the material parameters for numerical analysis are listed in Table 1.

Table 1. Model parameters.

\begin{tabular}{cccccc}
\hline$E(\mathrm{MPa})$ & $v$ & $\rho\left(\mathrm{kg} / \mathrm{m}^{3}\right)$ & $\phi\left(^{\circ}\right)$ & $\psi\left({ }^{\circ}\right)$ & $c(\mathrm{kPa})$ \\
\hline 80.0 & 0.25 & 1850 & 18 & 0 & 2.5 \\
\hline
\end{tabular}

Numerical results have been obtained for a series of strength reduction factor from 1.0 to 2.53 , and a safety factor of 2.53 is obtained for this slope. In Fig.1, the contour of displacement and equivalent plastic strain obtained for $F_{s}=2.53$ are displayed. The maximum displacement is obtained at the toe of the slope, which is typical. It can also be seen that for this value of the reduction factor, a shear band highlighted by the concentrated plastic strain has developed and penetrated the slope. This indicates that the slope is already at the limit state of losing stability. From the curve of the maximum displacement against the reduction factor, we can see that the maximum displacement increase significantly as the reduction factor exceeds the value of 2.5. For comparison, the displacement finite element analysis using software Abaqus and the finite element limit analysis for upper and lower bounds (Lyamin \& Sloan, 2002a, 2002b) using software OptumG2 have also been carried out. All the safety factors obtained from these different approaches lie in a narrow range from 2.52 to 2.54 . 

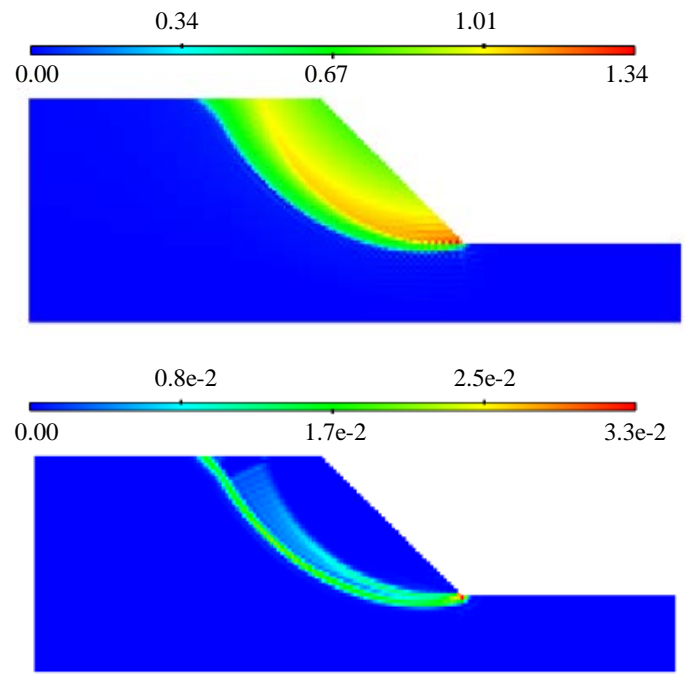

Fig. 1. Contour plots displacement (top) and equivalent plastic strain (bottom).

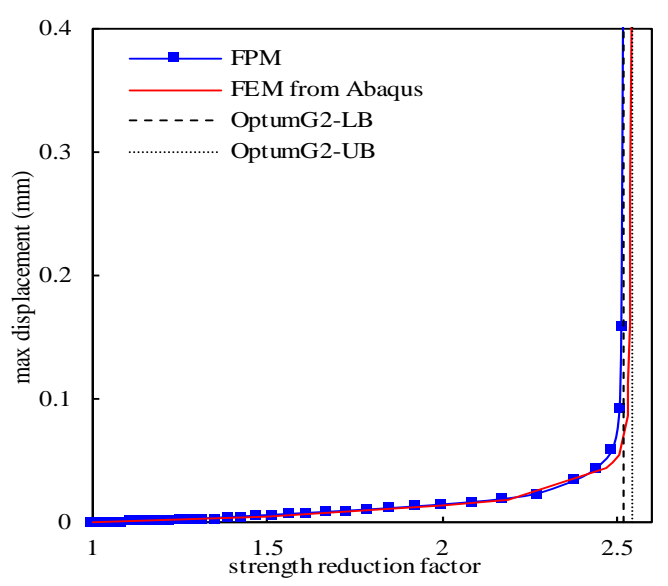

Fig. 2. The toe displacement against the reduction factor.

\section{CONCLUSIONS}

In the work, the FPM is formulated as equilibrium problems, rather than conventionally as dynamic problems. The method is thus suitable for solving large static deformation problems. The main numerical issues for implementation of the model, including solution method, hourglass control, tension instability elimination, stress integration for large deformation, have been discussed. For application of the method to stability analysis, the strength reduction method can be employed easily. The numerical results demonstrated the effective and accuracy of the method. The method has many potentials in analysis of large deformation and failure flow problems.

\section{ACKNOWLEDGEMENTS}

This work is financially supported by NSFC project (Grant No. 11772117/A020311), which is gratefully acknowledged.

\section{REFERENCES}

1) Lucy, L. B. (1977): A numerical approach to the testing of the fission hypothesis. The Astronomical Journal, 82(12), 1013-1024.

2) Monaghan, J. J. (1994): Simulating free surface flows with SPH. Journal of Computational Physics, 110(2), 399-406.

3) Chen, J. K., Beraun, J. E., \& Carney, T. C. (1999): A corrective smoothed particle method for boundary value problems in heat conduction. International Journal for Numerical Methods in Engineering, 46(2), 231-252.

4) Liu, M. B., \& Liu, G. R. (2006): Restoring particle consistency in smoothed particle hydrodynamics. Applied Numerical Mathematics, 56(1), 19-36.

5) Bonet, J., \& Kulasegaram, S. (2001): Remarks on tension instability of Eulerian and Lagrangian corrected smooth particle hydrodynamics (CSPH) methods. International Journal for Numerical Methods in Engineering, 52(11), 1203-1220.

6) Chen D., Huang W., Sloan S.W. (2019): An alternative updated Lagrangian formulation for finite particle method. Computer Methods in Applied Mechanics and. Engineering, 343, 490-505.

7) Ganzenmüller, G. C., Sauer, M., May, M., Hiermaier, S. (2016): Hourglass control for Smooth Particle Hydrodynamics removes tensile and rank-deficiency instabilities: Hourglass control for SPH. European Physical Journal: Special Topics, 225(2), 385-395.

8) Vidal, Y., Bonet, J., Huerta, A. (2007): Stabilized updated Lagrangian corrected SPH for explicit dynamic problems. International Journal for Numerical Methods in Engineering, 69(13), 2687-2710.

9) Hughes T.J.R. and Winget J. (1980): Finite rotation effects in numerical integration of rate constitutive equations arising in large deformation analysis. International Journal for Numerical Methods in Engineering, 15(12), 1862-1867.

10) Lyamin, A. V., \& Sloan, S. W. (2002): Lower bound limit analysis using non-linear programming. International Journal for Numerical Methods in Engineering, 55(5), 573-611.

11) Lyamin, A. V., \& Sloan, S. W. (2002): Upper bound limit analysis using linear finite elements and non-linear programming. International Journal for Numerical and Analytical Methods in Geomechanics, 26(2), 181-216. 